\title{
EFEITO DA DENSIDADE POPULACIONAL SOBRE AS CURVAS DE CRESCIMENTO DE MACROBRACHIUM ROSENBERGII (DE MAN) EM CULTIVO SEMI-INTENSIVO (CRUSTACEA, PALAEMONIDAE) ${ }^{1}$
}

\author{
Wagner Cotroni Valenti ${ }^{2}$ \\ Jeanette de Toledo Cardoso de Mello ${ }^{3}$ \\ Newton Castagnolli ${ }^{2}$
}

\begin{abstract}
THE EFFECT OF STOCKING DENSITY ON MACROBRACHIUM ROSENBERGII (DE MAN) GROWTH CURVES IN EARTHEN PONDS (CRUSTACEA, PALAEMONIDAE). In this article the length growth curves of Macrobrachium rosenbergii (De Man, 1879) held in different population densities were analysed. Postlarvae prawns were stocked into five 0,02 ha earthen ponds at densities of $4,8,12,16$ and 20 ind./ $\mathrm{m}^{2}$ during six months. Forthnightly, the mean total length of animals from each pond were obtained by means of random samples. For each population the length growth curves and the instantaneous growth rate expressions were determined. The growth rate and the asymptotic maximum length decreased with the stocking density raised. This effect is greater when the density ranges in low levels. The growth decrease can be due to intraspecific competition for life resources and to negative interactions between individuals, as aggressive or social behavior, that increase with the increase prawns density.

KEY WORDS. Crustacea, Palaemonidae, Macrobrachium rosenbergii, ecology
\end{abstract}

Macrobrachium rosenbergii (De Man, 1879) é conhecido popularmente como camarão da Malásia, gigante da Malásia ou camarão de água doce.

Diversos estudos referentes a aspectos de sua biologia foram realizados nas três últimas décadas tornando-se possível e viável economicamente seu cultivo em larga escala. Atualmente, esta é a espécie de camarão de água doce mais utilizada em projetos de carcinicultura em todo o mundo, sendo a única cultivada no Brasil com fins comerciais (VALENTI, no prelo).

$\mathrm{O}$ crescimento é um dos aspectos do desenvolvimento de um animal e pode ser expresso como o aumento em comprimento, volume ou peso ao longo do tempo (NIKOLSKII, 1969; HARTNOLL, 1982). Resulta de um balanço entre os processos de anabolismo e catabolismo que ocorrem no indivíduo (VON BERTALANFFY, 1938).

1) Trabalho financiado pela FAPESP.

2) Departamento de Biologia Aplicada, Universidade Estadual Paulista, 14870-000 Jaboticabal, São Paulo, Brasil.

3) Instituto de Biociências, Universidade de São Paulo, Caixa Postal 11461, 05422-970 São Paulo, São Paulo, Brasil. 
O estudo do crescimento é importante para o conhecimento da biologia de uma espécie e fundamental para o delineamento de projetos de aquicultura, pois este é um dos principais condicionadores da produtividade.

Tanto para aplicações práticas como teóricas, é interessante que o crescimento seja representando através de modelos matemáticos que o descrevam de forma generalizada (BEVERTON \& HOLT, 1957; BAGENAL, 1978; PAULY, 1980).

Assim, neste trabalho, foram determinadas as curvas de crescimento em comprimento de $M$. rosenbergii mantido em diferentes densidades populacionais, com o objetivo principal de fornecer subsídios para a otimização do cultivo desta espécie.

\section{MATERIAL E MÉTODOS}

Este trabalho foi desenvolvido no Setor de Carcinicultura da Faculdade de Ciências Agrárias e Veterinárias e Centro de Aquicultura da Universidade Estadual Paulista, Jaboticabal, São Paulo $\left(21^{\circ} 15^{\prime} 22^{\prime \prime}\right.$ S e $\left.42^{\circ} 18^{\prime} 48^{\prime \prime} W\right)$. A fase experimental foi conduzida no período de 9-XI-87 a 11-V-88.

Cinco viveiros escavados com área de $200 \mathrm{~m}^{2}$ cada um foram povoados com pós-larvas de $M$. rosenbergii nas densidades de 4, 8, 12, 16 e 20 ind./ $\mathrm{m}^{2}$.

Durante o período de cultivo os animais foram alimentados diariamente com ração peletizada com conteúdo protéico de $35 \%$. Os principais parâmetros da água de interesse para a carcinicultura foram monitorados e mantidos dentro da faixa adequada para o bom desenvolvimento da espécie de acordo com as recomendações de NEW \& SINGHOLKA (1985) e VALENTI (1985).

Quinzenalmente determinou-se o comprimento médio dos camarões presentes em cada viveiro, através de amostras aleatórias.

Geralmente, chama-se curva de crescimento em comprimento à relação entre uma medida de comprimento e a idade dos indivíduos. VON BERTALANFFY (1938) desenvolveu um modelo para expressar esta relação, com base em informações fisiológicas que tem se ajustado muito bem à grande maioria das espécies de peixes e crustáceos estudados e tem sido utilizado sob a forma:

$$
L_{t}=L_{\infty}\left[1-e^{-K\left(t-t_{0}\right)}\right]
$$

Onde, $\mathbf{L}_{\mathbf{t}}$ : comprimento médio dos indivíduos com idade "t"; $\mathbf{L} \infty$ : comprimento médio máximo assintótico que os indivíduos podem atingir; e: base dos logarítmos naturais; $\mathbf{K}$ : coeficiente de crescimento; $\mathbf{t}$ : idade dos indivíduos; to: parâmetro relacionado com o comprimento do animal à nascer, que pode ser considerado igual a zero quando este comprimento for desprezível em relação ao valor de "L $\infty "$.

Em cultivos intensivos ou semi-intensivos a idade dos indivíduos (t) pode ser decomposta da seguinte forma:

$$
t=T+t_{e}
$$


Onde, T: tempo de cultivo; $t_{\mathrm{e}}$ : idade dos indivíduos na data de estocagem. As condições ambientais e populacionais a que os animais são submetidos no período de pré-estocagem, geralmente são bastante diferentes daquelas do cultivo. Desse modo, o crescimento será expresso por equações matemáticas diferentes nestas duas fases. Na carcinicultura, deve-se considerar, ainda, a existência da fase larval que compreende um conjunto de estágios morfologicamente distintos e metamorfose, sendo muito provável que o padrão de crescimento seja alterado na passagem para a fase pós-larval, que é aquela utilizada no cultivo. Como decorrência, quando relaciona-se o comprimento à idade real, freqüentemente não se obtém um bom ajuste da curva teórica determinada aos dados experimentais. Deve-se destacar também que, em aquicultura, o objetivo principal é conhecer a variação do tamanho dos animais em função do tempo de cultivo ao invés da idade real.

Assim, para aplicação em aquicultura a expressão de VON BERTALANFFY pode ser escrita sob a forma:

$$
L_{T}=L_{\infty}\left(1-e^{-K T}\right)
$$

Sendo, LT:comprimento médio dos indivíduos no tempo de cultivo "T"; L: comprimento médio máximo assintótico que os indivíduos podem atingir nas condições de cultivo praticadas

A expressão acima foi ajustada para as cinco populações estudadas através do método interativo desenvolvido por E.P. SANTOS (com. pes.) utilizando-se o programa computacional denominado "CAJUS". Optou-se pelo uso deste método, porque as equações estimadas através dele mostraram melhor ajuste aos dados experimentais do que aquelas obtidas a partir do método de Ford-Walford (WALFORD, 1946), tradicionalmente utilizado para a estimativa dos parâmetros " $\mathrm{L} \infty$ " e "K".

A derivada da expressão de VON BERTALANFFY em relação ao tempo de cultivo resulta na equação que fornece a taxa de crescimento $\left(V_{T}\right)$, ou seja:

$$
V_{T}=\frac{d L_{T}}{d T}=K\left(L_{\infty}-L_{T}\right)
$$

Observa-se que a taxa instatânea de crescimento varia linearmente em função de " $\mathrm{LT}_{\mathrm{T}}$ de forma decrescente e depende dos parâmetros "L" e "K".

A partir das curvas de crescimento em comprimento previamente ajustadas obteve-se a expressão de $\mathrm{V}_{\mathrm{T}}$ para as cinco populações estudadas.

\section{RESULTADOS E DISCUSSÃO}

As curvas de crescimento em comprimento foram obtidas relacionandose o comprimento médio dos animais em cada quinzena $\left(\mathrm{L}_{\mathrm{T}}\right)$ ao tempo de cultivo ( $\mathrm{T}$ ) a partir de $\mathrm{T}=1$, ou seja, da primeira amostragem realizada. A 
tentativa de obtenção desta curva em função da idade real dos camarões mostrou que o modelo de VON BERTALANFFY não se ajusta aos dados experimentais. Do mesmo modo, a inclusão do comprimento médio dos animais na estocagem $(T=0)$ aos dados utilizados para a determinação da relação "LT/T" mostrou baixa aderência dos dados à curva resultante, caracterizando a incompatibilidade do modelo.

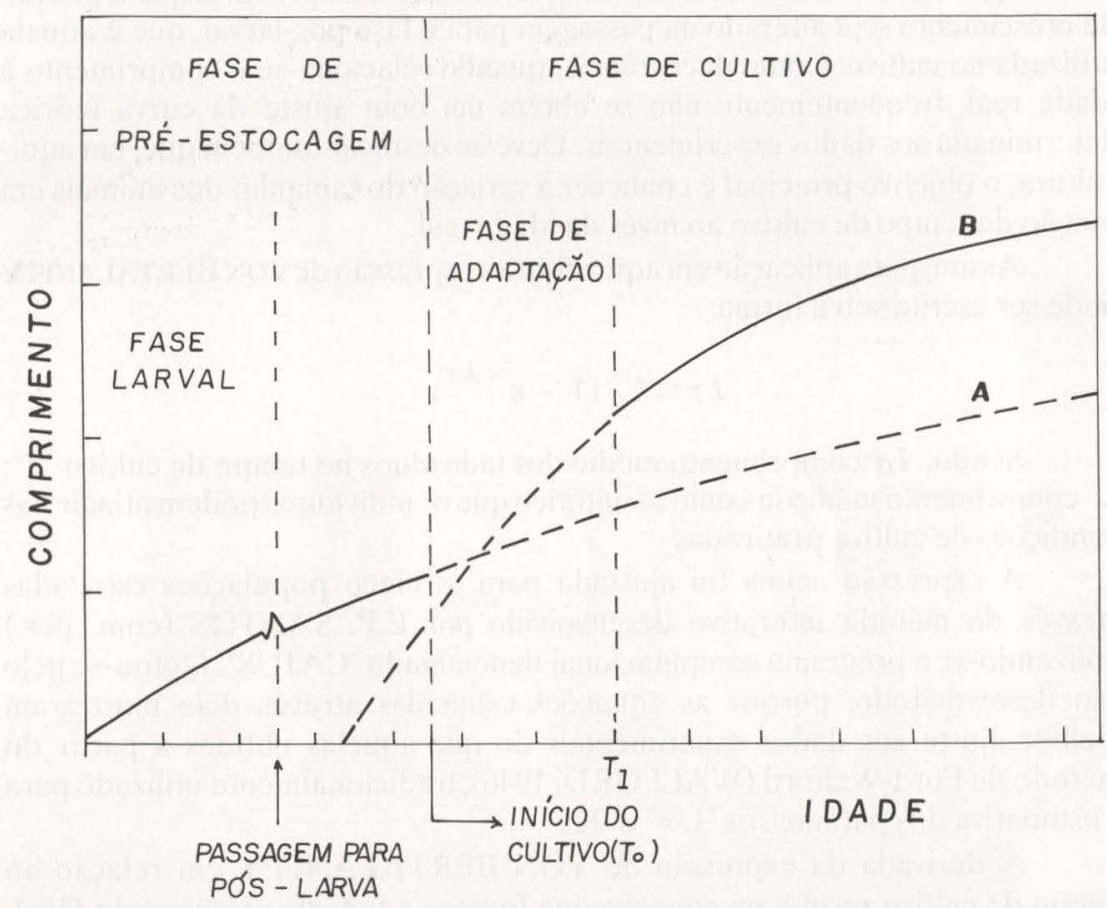

Fig. 1. Representação gráfica das possíveis modificações no padrão de crescimento dos camarões submetidos às condições de cultivo semi-intensivo. (A) Curva de crescimento em comprimento da fase pós-larval nas condições de pré-estocagem; (B) curva de crescimento em comprimento da fase pós-larval nas condições de cultivo.

No caso das populações utilizadas na carcinicultura, poderão ocorrer variações no modelo que descreve o crescimento dos animais ao longo de seu desenvolvimento ontogenético como ilustra a figura 1. Assim, o crescimento pode se alterar com a passagem da fase larval para a pós-larval devido a alterações metabólicas e com a passagem da fase de pré-estocagem para a fase de cultivo devido a alterações ambientais e populacionais. Após a estocagem nos viveiros, o animal poderá passar por um período de adaptação às novas condições, no qual o crescimento será afetado tanto pelos fatores atuantes na pré-estocagem como pelos atuais. Desse modo, os dados obtidos neste período de transição, geralmente, não estarão de acordo com o modelo de crescimento válido para a fase de cultivo (Curva B) nem com aquele válido para a pré- 
estocagem (Curva A). Isto explicaria porque, neste trabalho, só se obteve um bom ajuste da curva de crescimento em comprimento a partir do instante $\mathrm{T}_{1}$.

A ocorrência de alteração no modelo de crescimento com a passagem da fase de pré-estocagem para o cultivo foi observada no peixe Prochilodus cearensis Steindachner por PERET (1980).

$\mathrm{Na}$ figura 2 são apresentadas as curvas de crescimento em comprimento obtidas para as cinco densidades. As equações correspondentes são:

$\begin{array}{cr}\left.\text { Densidade (ind. } / \mathrm{m}^{2}\right) & \text { Curva de crescimento em comprim } \\ 4 & \mathrm{~L}_{\mathrm{T}}=218 \cdot\left(1-\mathrm{e}^{-0,100 \cdot \mathrm{T}}\right) \\ 8 & \mathrm{~L}_{\mathrm{T}}=162 \cdot\left(1-\mathrm{e}^{-0,138 \cdot \mathrm{T}}\right) \\ 12 & \mathrm{~L}_{\mathrm{T}}=119 \cdot\left(1-\mathrm{e}^{-0,183 \cdot \mathrm{T}}\right) \\ 16 & \mathrm{LT}_{\mathrm{T}}=119 \cdot\left(1-\mathrm{e}^{-0,175 \cdot \mathrm{T}}\right) \\ 20 & \mathrm{LT}_{\mathrm{T}}=108 \cdot\left(1-\mathrm{e}^{-0,171 \cdot \mathrm{T}}\right)\end{array}$

A variação das taxas de crescimento instantâneo em função de $\mathrm{L}_{\mathrm{T}}$ nas cinco populações estudadas é apresentada na figura 3 . As equações obtidas são:

Densidade (ind./ $\mathrm{m}^{2}$ )

4

8

12

16

20
Taxa de crescimento instantâneo

$$
\begin{aligned}
& \mathrm{V}_{\mathrm{T}}=21,8-0,100 . \mathrm{L}_{\mathrm{T}} \\
& \mathrm{V}_{\mathrm{T}}=22,4-0,138 . \mathrm{L}_{\mathrm{T}} \\
& \mathrm{V}_{\mathrm{T}}=21,8-0,183 . \mathrm{L}_{\mathrm{T}} \\
& \mathrm{V}_{\mathrm{T}}=20,8-0,175 . \mathrm{L}_{\mathrm{T}} \\
& \mathrm{V}_{\mathrm{T}}=18,5-0,171 . \mathrm{L}_{\mathrm{T}}
\end{aligned}
$$

As expressões acima indicam que o comprimento médio máximo assintótico atingido pelos camarões e a taxa instantânea de crescimento diminuem em função da densidade de estocagem (Figs 3 e 4).

A variação no crescimento foi relativamente pequena quando a densidade foi elevada de 12 a $20 \mathrm{ind} . / \mathrm{m}^{2}$, sendo que nas densidades de $12 \mathrm{a} 16 \mathrm{ind} . / \mathrm{m}^{2}$ os valores de $\mathrm{L}$ obtidos foram idênticos e as taxas de crescimento muito próximas. Isto sugere que o efeito da densidade da população estocada sobre o crescimento dos camarões seja mais acentuado quando esta varia em níveis mais baixos, de forma semelhante ao que foi observado em alguns experimentos realizados com peixes, revisados e discutidos por BACKIEL \& LE CREN (1978).

A redução da taxa média de crescimento de $M$. rosenbergii quando a densidade é aumentada foi constatada por alguns autores em diversos intervalos de variação da densidade de estocagem (SANDIFER \& SMITH, 1975; WILLIS 


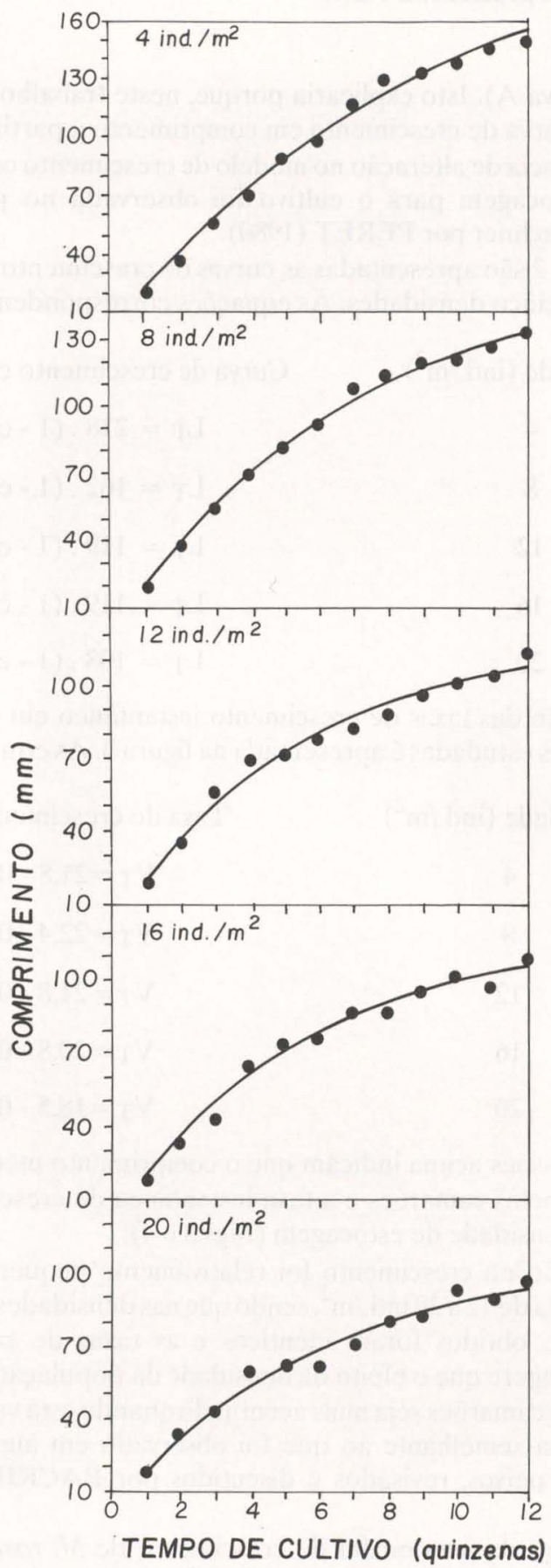

Fig. 2. Curvas de crescimento em comprimento obtidas para as cinco densidades estudadas. 
\& BERRIGAN, 1977; BRODY et al., 1980; SMITH et al., 1981; 1982; COHEN et al., 1981; ROUSE \& STICKNEY, 1982; KARPLUS et al., 1986). Nenhuma informação, foi encontrada referente ao tamanho médio máximo atingido pelos camarões.

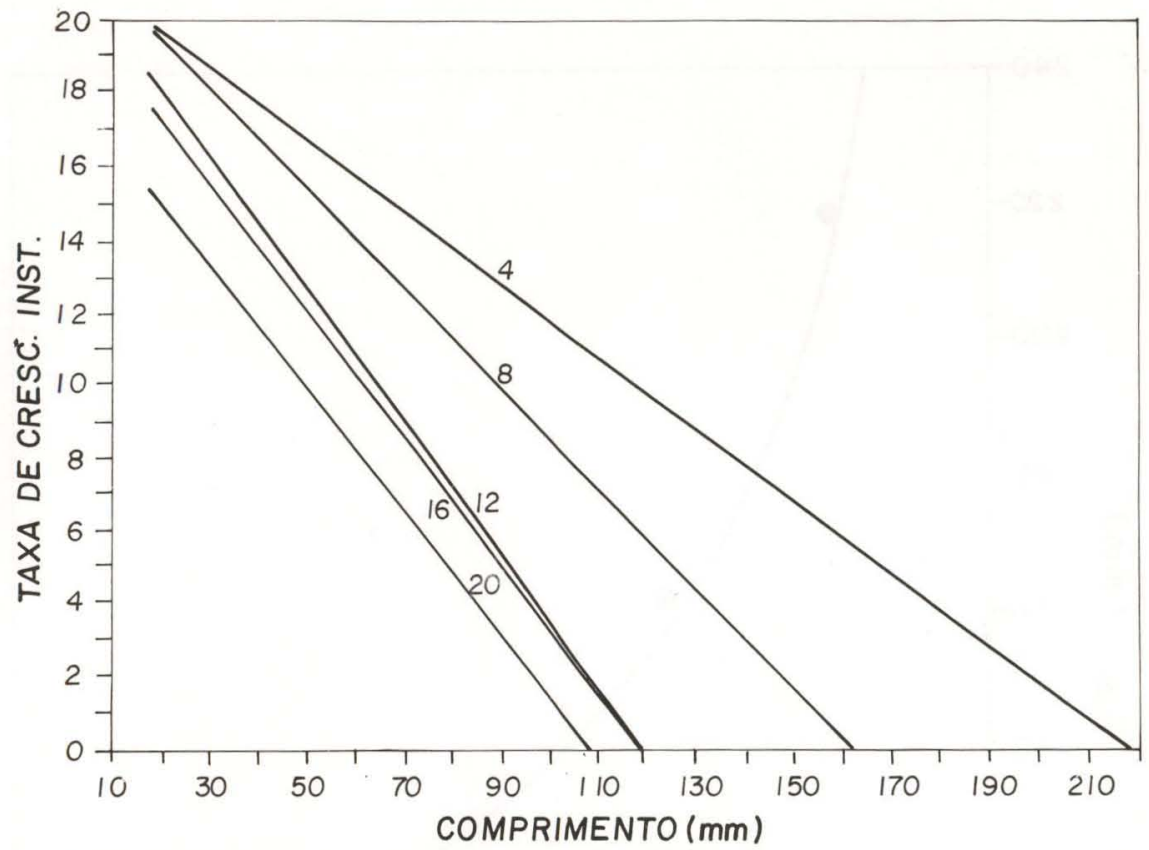

Fig. 3. Variação das taxas instantâneas de crescimento em função do comprimento dos indivíduos cultivados nas cinco densidades estudadas (números sobre as retas).

O crescimento de qualquer organismo depende tanto de fatores intrínsecos, próprios da constituição genética de cada população, como de fatores extrínsecos (ambientais) que agem sobre o indivíduo durante todo o seu desenvolvimento ontogenético. Segundo HARTNOLL (1982) os fatores exógenos que mais interferem no crescimento dos crustáceos são a temperatura e a disponibilidade de alimento.

Como as pós-larvas utilizadas na instalação deste experimento foram distribuídas aleatoriamente nos cinco viveiros é bastante improvável que fatores endógenos tenham produzido diferença entre os tratamentos. Os parâmetros físicos e químicos da água mantiveram uma variação semelhante nos cinco viveiros e, portanto, pouco devem ter interferido no crescimento dos camarões. Assim, os resultados obtidos devem ser realmente decorrentes das condições populacionais a que os animais foram submetidos, ou seja, as densidades de estocagem.

A elevação do tamanho da população no interior de um viveiro tem como efeito imediato o aumento da competição intraespecífica por recursos vitais, 
como alimento, espaço, etc. Quando um desses recursos tornar-se insuficiente para atender às necessidades de todos os indivíduos, passará a ser limitante e o crescimento poderá ser reduzido tanto pela carência do recurso como pelo maior gasto de energia motivado pelo estresse decorrente da competição.

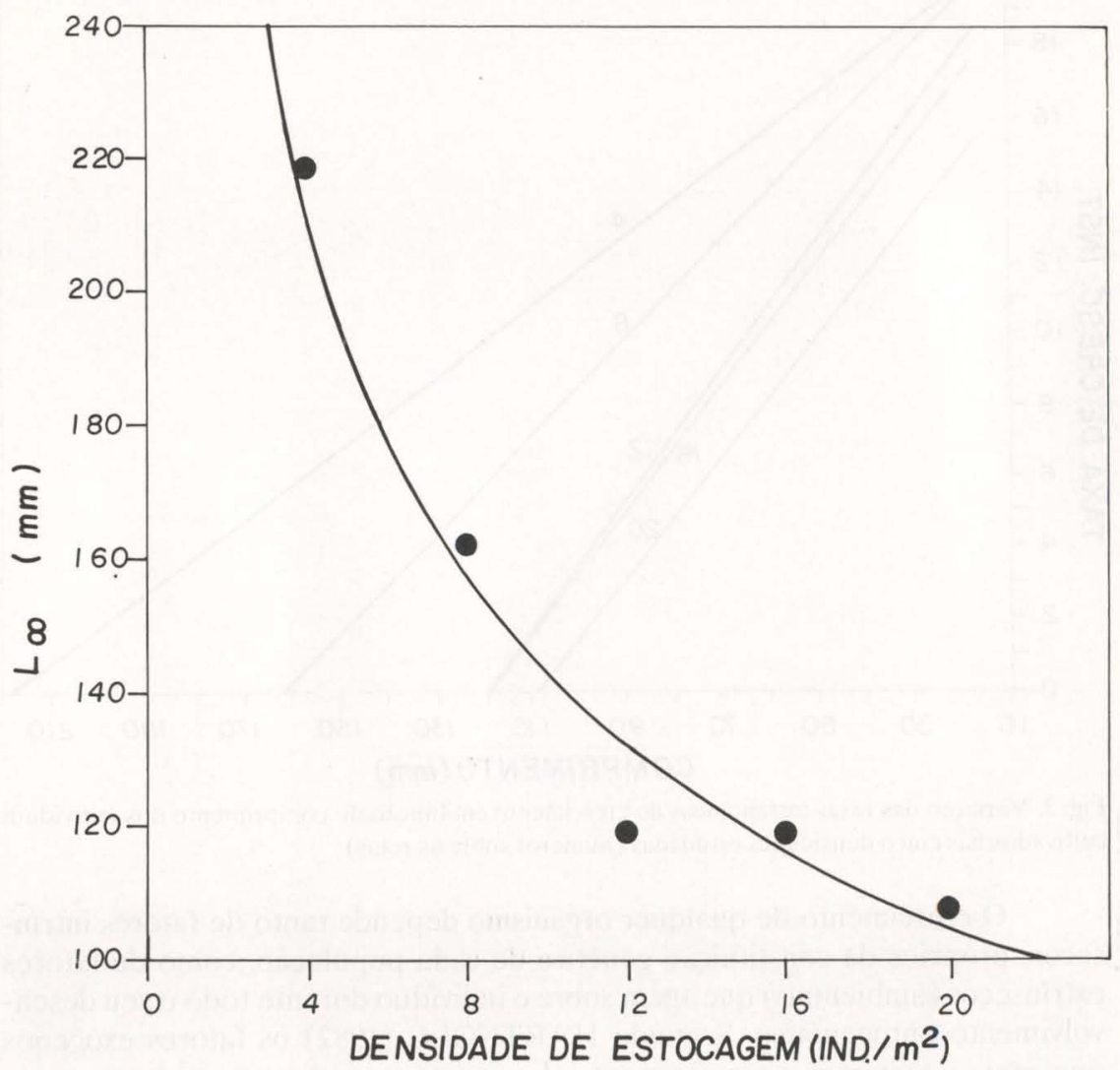

Fig. 4. Variação dos valores de comprimento médio máximo $(\mathrm{L})$ obtidos nas cinco densidades estudadas.

A quantidade de ração fornecida aos camarões foi proporcional à biomassa de cada viveiro. No entanto, existem fortes indícios de que $M$. rosenbergii depende fundamentalmente do alimento natural presente nos viveiros (SCHROEDER, 1983; SANDIFER \& SMITH, 1985, VALENTI, 1990). Sendo verdadeira esta afirmativa haveria um aumento na competição por alimento com a elevação da densidade de estocagem independentemente da quantidade de ração administrada.

É conhecida a ocorrência de comportamento territorial em M. rosenbergii (PEEBLES, 1979a; 1979b; COHEN et al., 1981). O gasto de energia decorrente 
da disputa por um território e da defesa do mesmo será certamente maior em densidades mais elevadas, podendo vir a contribuir para uma diminuição na taxa de crescimento. Realmente, COHEN et al. (1983) observaram um aumento significativo na taxa de crescimento de $M$. rosenbergii quando abrigos foram colocados no fundo dos viveiros para reduzir a competição por espaço.

Além da competição, outro fator que pode influir no crescimento dos camarões é a ação direta entre indivíduos através de comportamento agressivo ou social.

A agressividade individual, com a ocorrência, inclusive, de canibalismo é bem conhecida em camarões do gênero Macrobrachium (LING, 1969; PEEBLES, 1979a; 1979b; VALENTI, 1985). Desse modo, os animais de maior porte poderiam, mesmo havendo abundância de alimento, impedir ou dificultar a ingestão deste pelos menores. Segundo KREBS (1972) quando ocorre disputa por um recurso com agressividade entre os competidores o estresse decorrente da competição é intenso mesmo que o recurso seja abundante. A elevação da densidade populacional certamente aumentará a probabilidade de encontro dos camarões e, conseqüentemente, a manifestação do comportamento agressivo.

Sabe-se que existe em $M$. rosenbergii uma estrutura social definida, com três castas de machos: os portadores de quelípodos azul profundo (BC), que são grandes, dominantes, territoriais e férteis; os portadores de quelípodos alaranjados (OC), de tamanho semelhante aos BC, mas com baixa atividade gonadal e os portadores de quelípodos translúcidos (SM) que são bem menores que os anteriores, férteis e de comportamento mais ativo (COHEN et al., 1981; KURIS et al., 1987; SAGI \& RA'ANAN, 1988).

Os mecanismos de manutenção dessa estrutura social são pouco conhecidos. No entanto, COHEN et al. (1981) demonstraram que a proporção entre os machos pertencentes aos três morfotipos é aproximadamente constante em todas as populações e não é afetada pela densidade. Assim, fica afastada a hipótese de aumento da freqüência de indivíduos dominados quando a densidade é elevada.

A inibição do crescimento por ação direta entre indivíduos pode se dar ainda, através de ferormônios. Não foram encontradas na literatura, no entanto, informações que corroborem esta hipótese.

As três espécies de camarões de água doce nativas no Brasil de maior interesse econômico são $M$. amazonicum, M. acanthurus (Wiegmon, 1836) e $M$. carcinus Linnaeus, 1758 (COELHO et al., 1982; VALENTI, 1985). As curvas de crescimento em comprimento dessas espécies foram determinadas, respectivamente por GUEST (1979) em condições de cultivo em laboratório, VALENTI et al. (1987) e VALENTI (1984) na natureza. Comparando-se essas curvas com aquelas obtidas neste trabalho para $M$. rosenbergii observa-se que a taxa de crescimento desta última espécie é bastante superior. $O$ tamanho médio máximo atingido por $M$. rosenbergii é próximo daquele atingido por $M$. carcinus e bem maior que o atingido por $M$. acanthurus e $M$. amazonicum.

Estas informações indicam que, quanto ao crescimento, M. rosenbergii é 
mais adequado ao cultivo que as espécies acima citadas. No entanto, estas podem apresentar vantagens em outras características também importantes para a carcinicultura, como por exemplo maior resistência às baixas temperaturas e melhor adaptação às condições do ambiente brasileiro, ainda não reveladas devido à falta de pesquisas adequadas.

Finalmente, deve-se destacar que, em animais que apresentam um exoesqueleto rígido, como os crustáceos, o crescimento é um processo essencialmente descontínuo. Existe uma sucessão de mudas separadas por períodos de intermudas e a quase totalidade do crescimento ocorre imediatamente após as mudas antes do endurecimento do tegumento novo. Após esta fase, durante a intermuda, pode ocorrer um pequeno crescimento devido à flexibilidade das membranas que ligam as placas que compõem o esqueleto rígido (MAUCHLINE, 1977; HARTNOLL, 1982). Assim, as curvas de crescimento ajustadas neste trabalho para $M$. rosenbergii representam, de forma contínua, a somação deste processo.

AGRADECIMENTOS. Ao Prof. Dr. Edison Pereira dos Santos por ter autorizado a utilização do programa computacional "CAJUS" de sua autoria, bem como pelas sugestões apresentadas ao longo do desenvolvimento deste trabalho.

\section{REFERÊNCIAS BIBLIOGRÁFICAS}

BACKIEL, T. \& E.D. LE CREEN. 1978. Some density relationships for fish populations parameters, p. 279-302. In: S.D. GERKING (ed.). Ecology of freshwater fish production. Oxford, Blackwell Scientific Publications.

BAGENAL, T.B. 1978. Methods for assessment of fish production in freshwaters. Oxford, Blackwell Scientific Publications, 3th ed., 365p.

BERTALANFFY, L. VON. 1938. A quantitative theory of organic growth. Hum. Biol., Baltimore, 10: 181-213.

BEVERTON, R.J.H. \& S.J. HOLT. 1957. On the dynamics of exploited fish population. Fishery Invest. Lond., Ser. 2, 19: 1-533.

BRODY, T.; D. COHEN; A. BARNES \& A. SPECTOR 1980. Yield characteristics of the prawn Macrobrachium rosenbergii in temperate zone aquaculture. Aquaculture, Amsterdam, 21: 375-85.

COELHO, P.A.; M. RAMOS-PORTO \& C.M.A. SOARES 1982. Biologia e cultivo de camarões de água doce. Série Aquicultura, v.1, Centro de Tecnologia, Departamento de Oceanografia. Recife, Universidade Federal de Pernambuco, 53p.

COHEN, D.; Z. RA'ANAN \& T. BRODY 1981. Population profile development and morphotypic differentiation in the giant freshwater prawn Macrobrachium rosenbergii (De Man). Journ. World Mariculture Soc. 12 (2): 231-43.

COHEN, D.; Z. RA'ANAN; V. RAPPAPORT \& Y. ARIELI 1983. The production of the freshwater prawn Macrobrachium rosenbergii (De Man) in 
Israel: Improved conditions for intensive monoculture. Bamidgeh, NirDavid, 35 (2): 31-7.

GUEST, W.C. 1979. Laboratory life history of the palaemonid shrimp Macrobrachium amazonicum (Heller) (Decapoda, Palaemonidae). Crustaceana, Leiden, 37 (2): 141-52.

HARTNOLL, R.G. 1982. Growth, p.111-196. In: D.E. BLISS (ed.). The Biology of Crustacea, Embriology, Morphology and Genetics. New York, Academic Press., v.2.

KARPLUS, I.; G. HULATA; G.W. WOHLFARTH \& A. HALEVY.1986. The effect of density of Macrobrachium rosenbergii raised in earthen ponds on their population structure and weight distribution. Aquaculture, Amsterdam, 52: 307-20.

KREBS, C.J. 1972. Ecology: The experimental analysis of distribuition and abundance. New York, Harper \& Row., 694p.

KURIS; A.M.; Z. RA'ANAN; A. SAGI \& D. COHEN. 1987. Morphotypic differentiation of male malaysian giant prawns Macrobrachium rosenbergii. Journ. Crust. Biol., Woods Hole, 7 (2): 219-37.

LING, S.W. 1969. The general biology and development of Macrobrachium rosenbergii (De Man). FAO Fish. Rep., Rome, 3 (57): 589-606.

MAUCHLINE, J. 1977. Growth of shrimps, crabs and lobsters: an assessment. J. Cons. int. Explor. Mer., Copenhague, 37 (2): 162-9.

NEW, M.B. \& S. SINGHOLKA 1985. Freshwater prawn farming. A manual for the culture of Macrobrachium rosenbergii. FAO Fish. Tec. Pap., Rome, 225: $116 \mathrm{p}$.

NIKOLSKII, G.V. 1969. Theory of fish population dynamics as the biological background for rational exploitation and management of fishery resources. Edimbug, Oliver \& Boyd., 323p.

PAULY, D. 1980. A selection of simple methods for the assessment of tropical fish stocks. FAO Fish. Circ., Rome, 729: 54p.

PEEBLES, J.B. 1979a. Molting, movement, and dispersion in the freshwater prawn Macrobrachium rosenbergii. J. Fish. Res. Bd. Can. 36: 1080-8.

. 1979b. The roles of prior residence and relative size in competition for shelter by the malaysian prawn, Macrobrachium rosenbergii. Fish. Bull., Miami, 76 (4): 173-80.

PERET, A.C. 1980. Aspectos da influência da densidade populacional em cultivo intensivo com curimatã comum Prochilodus cearensis Steindachner (Characidae - Prochilodinae). Tese de Mestrado, não publicada, Universidade Federal de São Carlos, São Carlos, 87p.

ROUSE, D.B.\& R.R.STICKNEY.1982. Evaluation of the production potential of Macrobrachium rosenbergii in monoculture and polyculture with Tilapia aurea. Journ. World Mariculture Soc. 13: 73-85.

SAGI, A. \& Z. RA'ANAN. 1988. Morphotypic differentiation of males of the freshwater prawn Macrobrachium rosenbergii: changes in the midgut glands and the reproductive system. Journ. Crust. Biol., Woods Hole, 8 (1): 43-7. 
SANDIFER, P.A.\& T.I.J.SMITH 1975. Effects of population density on growth and survival of Macrobrachium rosenbergii reared in recirculating water management systems. Proc. World Maric. Soc. 6: 43-54.

- 1985. Freshwater prawns. In: HUNER, J.V. \& E.E. BROWN (ed.). Crustacean and Mollusk Aquaculture in the United States. Connecticut, AVI Publishing, 476p.

SCHROEDER, G.L. 1983. Sources of fish and prawn growth in polyculture ponds as indicated by $\mathrm{C}$ analysis. Aquaculture, Amsterdam, 35: 29-42.

SMITH, T.I.J.; P.A. SANDIFER \& W.E. JENKINS. 1982. Pond rearing trials with malaysian prawns, Macrobrachium rosenbergii by private growers in South Carolina, 1981. Journ. World Mariculture Soc. 13: 41-55.

SMITH, T.I.J.; P.A. SANDIFER; W.E. JENKINS \& A.D. STOKES. 1981. Effect of population structure and density of prawn (Macrobrachium rosenbergii) farming in temperate climates. Journ. World Mariculture Soc. 12 (1): 233-50.

VALENTI, W.C. 1984. Estudo Populacional dos Camarões de Água Doce Macrobrachium acanthurus (Wiegmann, 1836) e Macrobrachium carcinus (Linnaeus, 1758) do Rio Ribeira de Iguape (Crustacea, Palaemonidae). Tese de Mestrado, não publicada, Universidade de São Paulo, São Paulo, 149p.

. 1985. Cultivo de Camarões de Água Doce. São Paulo, Nobel. 82p.

. 1990. Criação de Camarões de Água Doce (Macrobrachium rosenbergii). Anais da $27^{\text {a }}$ Reuniáo anual da Sociedade Brasileira de Zootecnia e 12 ${ }^{\text {a }}$ Reunião da Associação Latino-americana de Produção Animal, Campinas, p.757-785.

- (no prelo). Current status of freshwater prawn farming in Brasil. Proceedings of the National Symposium on Freshwater Prawns (Macrobrachium sp.). Cochin, India.

VALENTI, W.C.; J.T.C. MELLO \& V.L. LOBÃO. 1987. Crescimento de Macrobrachium acanthurus (Wiegmann, 1836) do Rio Ribeira de Iguape (Crustacea, Decapoda, Palaemonidae). Revta Bras. Biol., Rio de Janeiro, 47 (3): 349-55.

WALFORD, L.A. 1946. A new grafic method of discribing the growth of animals. Biol. Bull. mar. biol. lab., Hoods Hole, 90 (2): 141-7.

WILLIS, A. \& E. BERRIGAN 1977. Growth of the malaysian prawn, Macrobrachium rosenbergii in the earthen ponds in the central Flórida. Completion Report for NOAA-NMFS, PL 88-309 project.

Recebido em 30.IX.1991; aceito em 25.XI.1993. 